\title{
In Vitro and In Vivo Effect of Xylopic Acid on Cytochrome P450 Enzymes
}

\author{
Mary A. Agbenyeku $\mathbb{D}^{1,2}$, Regina Appiah-Opong ${ }^{1}{ }^{1},{ }^{1}$ Ernest Obese ${ }^{(D)},{ }^{3}$ Robert P. Biney $\left(\mathbb{D},{ }^{3}\right.$ \\ Emmanuel A. Adakudugu $\left(\mathbb{D},{ }^{3}\right.$ Arnold D. Forkuo ${ }^{(D)}{ }^{4}$ Silas A. Osei $\mathbb{D}^{2}{ }^{2}$ \\ Mustapha K. Abeka $\mathbb{D}^{3}{ }^{3}$ and Elvis O. Ameyaw $\mathbb{D D}^{3}$ \\ ${ }^{1}$ Noguchi Memorial Institute for Medical Research, Department of Clinical Pathology, University of Legon, Accra, Ghana \\ ${ }^{2}$ Department of Biomedical Science, School of Allied Health Sciences, College of Health and Allied Sciences, \\ University of Cape Coast, Cape Coast, Ghana \\ ${ }^{3}$ School of Pharmacy and Pharmaceutical Sciences, College of Health and Allied Sciences, University of Cape Coast, Ghana \\ ${ }^{4}$ Faculty of Pharmacy and Pharmaceutical Sciences, College of Health Sciences, \\ Kwame Nkrumah University of Science and Technology, Kumasi, Ghana \\ Correspondence should be addressed to Elvis O. Ameyaw; eameyaw@ucc.edu.gh
}

Received 23 June 2021; Accepted 14 December 2021; Published 21 January 2022

Academic Editor: Mahmoud S. Ahmed

Copyright (c) 2022 Mary A. Agbenyeku et al. This is an open access article distributed under the Creative Commons Attribution License, which permits unrestricted use, distribution, and reproduction in any medium, provided the original work is properly cited.

\begin{abstract}
Introduction. Xylopic acid (XA), the major constituent of the fruit of Xylopia aethiopica, has shown several pharmacological properties. Traditionally, the plant is used to treat several diseases and is being used in the preparation of several local foods despite the lack of information about its safety, food-drug interaction, and other pharmacokinetic properties. This study, therefore, investigated the effect of XA on rat liver cytochrome P450 (CYP) enzymes in vivo and in vitro. Methods. Inhibition or induction of some isoforms of CYP450 enzymes: CYP 1A1/1A2, 1A2, 2B1/2B2, 3A4, 2D6, and 2C9 were investigated using microsomal fractions of the liver obtained from rats pretreated with a low dose of xylopic acid (LDT) $30 \mathrm{mg} / \mathrm{kg}$, high dose of xylopic acid (HDT) $100 \mathrm{mg} / \mathrm{kg}$, phenobarbitone (PC) $80 \mathrm{mg} / \mathrm{kg}$, and ketoconazole (NC) $100 \mathrm{mg} / \mathrm{kg}$, and a no-treatment group received distilled water, with $(n=5)$ animals in each group. The in vitro inhibition of CYP $3 \mathrm{~A} 4$ was assessed by treating rat liver microsomes with XA. Results. Xylopic acid induced CYP 1A1/1A2, 1A2, 2D6, and 2C9, inhibited CYP 3A4, and had no effect on 2B1/2B2. Conclusion. The findings would help mitigate toxicity and therapeutic failure especially in cases of coadministration of medications with food containing XA, with metabolism altered by the latter.
\end{abstract}

\section{Introduction}

The use of medicinal natural products is traced back thousands of years due to the belief that herbal drugs have fewer side effects, enhance the effects of conventional agents, and can be an alternative for the management of several disorders $[1,2]$. An example of one of such herbs is the Xylopia aethiopica. Studies have shown that the plant has anti-inflammatory, antimicrobial, analgesic, antiparasitic, and antioxidant properties [3-5]. In Ghana, the fruit is used by traditionalists to manage a wide range of disorders as it is believed to have curative effects on dyspepsia, cough, pain, and parasitic infections. It is equally used as a spice in the preparation of dishes such as porridge (Hausa Koko), sobolo, other African cuisines, and soups [5, 6]. Some food industries use it as a flavoring agent, and in some cosmetic industries, it is used as an agent for fragrance [7]. The plant is widely distributed in the West African rainforest from Senegal to Sudan in Eastern Africa and down to Angola in Southern Africa $[8,9]$ where it is mostly used for local cooking, especially in the preparation of what is referred to as the African pepper soup [10]. The plant grows prominently in several parts of Africa including Ghana, Nigeria, Benin, Kenya, Ivory Coast, and several other places [11]. Several studies have been performed on the fruit, and these studies reveal the plant contains about 98 compounds 
$[12,13]$. Constituents of the fruit include essential oils, volatile oils, resin, arocene, rutheroside fat, bitter principles, alkaloids, glycosides, saponins, tannins, mucilage, xylopic acid, and kaurenoic acid [14]. The compounds obtained from the fruit that are considered most relevant are xylopic acid and kaurenoic acid. Xylopic acid, a kaurene diterpene, occurs as the major constituent in the fruits of Xylopia aethiopica [15].

Research on xylopic acid has shown that it exhibits diuretic and vasorelaxant properties in rodents [16]. Xylopic acid caused a substantial reduction of lung inflammation induced by carrageenan in mice, which supports the traditional use of the plant extracts of Xylopia aethiopica as therapeutic agents in conditions associated with acute inflammation and some respiratory disorders [17]. Xylopic acid possesses curative and prophylactic properties on $P$. berghei-induced malaria in ICR mice as well as antipyretic properties [18]. Xylopic acid and the fruit extract of Xylopia aethiopica were found to have significant central nervous system depressant effects in mice $[3,19]$. The chemical structure of xylopic acid is shown in Figure 1.

Generally, people use traditional medicine with the notion that it has little or no side effects. Hence, such herbs do not pass through rigorous tests before approval is granted compared to conventional drugs $[20,21]$. Due to insufficient scrutiny, most herbal medicines may result in CYP-mediated herb-drug interactions when coadministered with other orthodox medications, and this may go unnoticed [22]. Interactions of traditional medicines with human CYPs have been associated with alterations in the pharmacokinetics of several drugs [23]. They interact by either inhibiting or inducing CYPs which may result in harmful side effects. Drug-drug and herb-drug interactions is an important phenomenon that should be investigated before new agents are introduced on the market. Hence, it is useful to conduct series of investigations on the effect of traditional medicines on liver CYP450 drug-metabolizing enzymes [20]. This study sought to evaluate the effect of xylopic acid on carefully selected CYPs that form the major drug-metabolizing enzymes in the human system.

\section{Materials and Methods}

2.1. Drugs and Chemicals. Phenobarbitone, ketoconazole, CYP450 isoenzymes, potassium phosphate dibasic, potassium phosphate monobasic, bovine serum albumin (BSA), nicotinamide adenine dinucleotide phosphate (NADPH), ethoxyresorufin, methoxyresorufin, pentoxyresorufin, benzyloxyresorufin, dextromethorphan, diclofenac, zinc sulphate heptahydrate, trimethylamine, and acetonitrile used to evaluate the effect of xylopic acid on CYPs were all obtained from Sigma-Aldrich Chemie GmbH (Eschenstrasse), Germany. XA was purified following the procedure described by Biney et al. [24].

2.2. Experimental Animals. Before the experiment, male Sprague-Dawley rats were purchased from the Animal Experimentation Unit of Noguchi Memorial Institute for

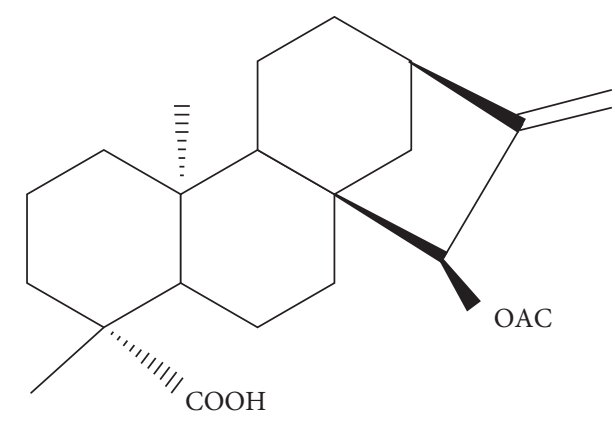

Figure 1: Chemical structure of xylopic acid [5].

Medical Research, Accra, Ghana and were fed ad libitum using standard animal laboratory pellet and water. They were housed under standard laboratory conditions of $25 \pm 1^{\circ} \mathrm{C}$ ambient temperature, $60-70 \%$ relative humidity, and $12: 12$ hour light: dark cycle to ensure acclimatization to the laboratory. The experiments were conducted following the National Institute of Health (NIH) Guidelines for Care and Use of Laboratory Animals and approved by the Scientific and Technical Committee and Institutional Animal Care and Use Committee (IACUC) of Noguchi Memorial Institute for Medical Research, University of Ghana (UGIACUC004/18-19).

2.3. In Vivo CYP450 Isoenzyme Induction Assay. The activities of xylopic acid high dose $(100 \mathrm{mg} / \mathrm{kg})$ and xylopic acid low dose $(30 \mathrm{mg} / \mathrm{kg})$ were tested on CYP 1A1/1A2 (EROD), CYP 2B1/2B2 (PROD), CYP 3A4 (BROD), CYP 2D6, and CYP 2C9 employing in vivo and in vitro assays as earlier described [25]. Ketoconazole, sulfaphenazole, diclofenac, dextromethorphan, and phenobarbitone were used as standard drugs in the study.

Male rats weighing 250-300 g $(n=5)$ were randomly assigned to one of the four experimental groups, being lowdose xylopic acid treatment group (LDT) $30 \mathrm{mg} / \mathrm{kg}$, highdose xylopic acid treatment group (HDT) $100 \mathrm{mg} / \mathrm{kg}$, phenobarbitone (PC) $80 \mathrm{mg} / \mathrm{kg}$, a no-treatment group that received distilled water, and finally for CYP 3A4, a control group treated with ketoconazole (KC) $100 \mathrm{mg} / \mathrm{kg}$ with $(n=5)$ animals in each group. The choice of dosage for XA was informed by previous studies on the interaction of XA on hepatic enzymes using pentobarbitone-induced sleep test in mice [24]. The treatment for each group was administered orally for 7 days. All the animals were anesthetized by $\mathrm{CO}_{2}$ inhalation to minimize suffering, then sacrificed by abdominal venesection method after which their liver was harvested, snap-frozen in liquid nitrogen, and stored at $-80^{\circ} \mathrm{C}$ until further analysis was carried out.

2.4. Preparation of Microsomal, Cytosolic Fractions. Liver samples of $7.5 \mathrm{~g}$ were obtained from each animal and kept in the $-80^{\circ} \mathrm{C}$ freezer until use. On the day of the experiment, each liver sample was then homogenized in two volumes of $20 \mathrm{mM}$ potassium phosphate buffer ( $\mathrm{pH}$ 7.4) using a mortar and a pestle. The homogenate was centrifuged at 2,500 rpm 
for 50 minutes at $4^{\circ} \mathrm{C}$ and further centrifuged at $40,000 \mathrm{rpm}$ for 60 minutes at $4^{\circ} \mathrm{C}$. The resultant supernatant (cytosol) was then separated from the pellet (microsomes). The microsomes obtained were further homogenized in potassium phosphate buffer $20 \mathrm{mM}\left(\mathrm{pH} \mathrm{7.4)}\right.$ and stored at $-80^{\circ} \mathrm{C}$ $[25,26]$.

2.5. Protein Determination and Standardization. The protocol for protein determination was adapted from the protocol described by Harwood et al. [25, 26]. Fourfold serial dilutions were performed on the microsomal solutions using potassium phosphate buffer. Then, a twofold serial dilution for seven concentrations was prepared for a protein standard, bovine serum albumin (BSA). A volume of $10 \mu \mathrm{L}$ of BSA and $200 \mu \mathrm{L}$ of Bio-Rad reagent which was then added to each microsomal dilution in a 96-well plate in triplicate and incubated at room temperature for 5 minutes, after which the absorbance was read at a wavelength of $530 \mathrm{~nm}$ excitation and $586 \mathrm{~nm}$ emission using Tecan Infinite M200 PRO.

2.6. The Effect of XA on CYP 1A1/1A2, 1A2, 3A4, and 2B1/2B2 Enzymes. The effect of xylopic acid on CYP 1A1/1A2, 1A2, $2 \mathrm{~B} 1 / 2 \mathrm{~B} 2$, and $3 \mathrm{~A} 4$ was determined using fluorometric assays as described previously [19]. This was achieved by pipetting $70 \mu \mathrm{L}$ of potassium phosphate buffer at a $\mathrm{pH}$ of 7.4 in triplicate into a 96-well plate followed by addition of $10 \mu \mathrm{L}$ $(2 \mu \mathrm{M})$ of each substrate (ethoxyresorufin (EROD), methoxyresorufin (MROD), pentoxyresorufin (PROD), and benzyloxyresorufin (BROD)). Subsequently, $10 \mu \mathrm{L}$ of the microsomal fraction obtained from each treatment group was added to each well plate and preincubated at $37^{\circ} \mathrm{C}$ for 5 minutes, after which, $10 \mu \mathrm{L}$ of nicotinamide adenine dinucleotide phosphate (NADPH) with a concentration of $100 \mu \mathrm{M}$ was added to each of the wells and incubated at $37^{\circ} \mathrm{C}$ for 10, 20, and 30 minutes for CYPs $1 \mathrm{~A} 1 / 1 \mathrm{~A} 2,1 \mathrm{~A} 2,2 \mathrm{~B} 1 / 2 \mathrm{~B} 2$, and $3 \mathrm{~A} 4$, respectively. Plates were gently shaken after the addition of $40 \mu \mathrm{L}$ of stopping solution (20\% $0.5 \mathrm{M}$ Tris and $80 \%$ acetonitrile) to each well. Fluorescence was determined at wavelengths of $586 \mathrm{~nm}$ emission and $530 \mathrm{~nm}$ excitation proceeding optimization of the protocol. The assay was repeated, and the mean values were used for computation.

2.7. In vitro Inhibitory Activity of XA on CYP $3 A 4$. Microsomes obtained from male rats pretreated with phenobarbitone were used in this assay. The standard inhibitor used was ketoconazole for CYP 3A4 and xylopic acid as the test compound. A cocktail was prepared for seven different concentrations for each standard inhibitor. The cocktail contained $3000 \mu \mathrm{l}$ of $0.1 \mathrm{M}$ potassium phosphate buffer $(\mathrm{pH}$ 7.4) which was pipetted into a $50 \mathrm{ml}$ Eppendorf tube, followed by the addition of $600 \mu$ l of the substrate (benzyloxyresorufin) for CYP 3A4, and followed by the addition of $600 \mu \mathrm{l}$ of the enzyme (microsomes). A volume of $80 \mu \mathrm{l}$ was then pipetted from the cocktail into a 96-well plate in triplicate and then preincubated at $37^{\circ} \mathrm{C}$ for 5 minutes. Afterward, $10 \mu \mathrm{L}$ of nicotinamide adenine dinucleotide phosphate (NADPH) with a concentration of $(100 \mu \mathrm{M})$ was added to each of the wells followed by the addition of ketoconazole and xylopic acid to separate plates containing BROD. The plates were incubated at $37^{\circ} \mathrm{C}$ for 30 minutes for BROD. A volume of $40 \mu \mathrm{L}$ of stopping solution $(20 \% 0.5 \mathrm{M}$ Tris and $80 \%$ acetonitrile) was finally added to each well. The plate was gently shaken, and its fluorescence read at wavelengths of $586 \mathrm{~nm}$ emission and $530 \mathrm{~nm}$ excitation [19]. The assay was repeated, and the mean values were used for computation.

2.8. Effect of XA on CYP 2D6. The CYP 2D6-dextromethorphan-O-demethylation assay was used to determine the effect of xylopic acid on CYP 2D6. The assay was done by pipetting $350 \mu \mathrm{L}$ of potassium phosphate buffer ( $\mathrm{pH} 7.4$ ) into Eppendorf tubes in triplicate. A volume of $50 \mu \mathrm{L}$ of $0.25 \mathrm{mM}$ dextromethorphan was added, followed by $50 \mu \mathrm{L}$ of microsomes obtained from each treatment group. The mixture was preincubated at $37^{\circ} \mathrm{C}$ for 5 minutes in a water bath after which $50 \mu \mathrm{L}$ of NADPH solution $100 \mu \mathrm{M}$ was added. The mixture was further incubated for 45 minutes, followed by the addition of $100 \mu \mathrm{L}$ of stopping solution $(300 \mathrm{mM}$ zinc sulphate heptahydrate). The mixture was centrifuged at $4,000 \mathrm{rpm}$ for $15 \mathrm{~min}$ at room temperature, and the supernatant was collected in vials. Analysis of the supernatant was done using an isocratic HPLC (Shimadzu Prominence, Japan). The mobile phase consisted of $24 \%$ (v/v) acetonitrile and $0.1 \%(\mathrm{v} / \mathrm{v})$ trimethylamine adjusted to $\mathrm{pH} 3.0$ with perchloric acid. The carrier flow rate was $0.8 \mathrm{ml} / \mathrm{min}$, and peaks were monitored at wavelengths of $280 \mathrm{~nm}$ excitation and $310 \mathrm{~nm}$ emission [19]. The assay was repeated, and the mean values were used for computation.

2.9. Effect of XA on CYP 2C9. The CYP 2C9 diclofenac hydroxylation assay was used to determine the effect of xylopic acid on CYP 2C9 [26]. This assay measures the effect of XA on hydroxylation of diclofenac to 4-hydroxydiclofenac by CYP 2C9. This was done by pipetting $350 \mu \mathrm{L}$ of potassium phosphate buffer $(\mathrm{pH}$ 7.4) into Eppendorf tubes followed by adding $50 \mu \mathrm{L}$ of $0.05 \mathrm{mM}$ diclofenac, after which $50 \mu \mathrm{L}$ of the microsomal fraction obtained from each treatment group was added (in triplicate) and preincubated at $37^{\circ} \mathrm{C}$ for 5 minutes in a water bath. A volume of $50 \mu \mathrm{L}$ of NADPH solution $(100 \mu \mathrm{M})$ was added to each tube and further incubated in a water bath at $37^{\circ} \mathrm{C}$ for 10 minutes. The reaction was terminated by adding $200 \mu \mathrm{L}$ of stopping solution (ice-cold methanol) to the mixture. The mixture was centrifuged at $12,000 \mathrm{rpm}$ for 8 minutes at room temperature. The supernatants were collected in vials and analyzed using high-performance liquid chromatography [15]. The assay was repeated, and the mean values were used for computation.

2.10. Data Analysis. For the in vivo testing, GraphPad Prism version 8 was used for all statistical analyses, and $P$ values $<0.05$ were considered statistically significant. All data obtained were expressed as a mean \pm standard error of the mean. For the in vitro analysis, the column graphs were 
subjected to one-way ANOVA with Tukey's post hoc test. Doses for $50 \%$ of the maximal effect $\left(\mathrm{IC}_{50}\right)$ for each drug were determined by using an iterative computer least squares method, with the following nonlinear regression (3parameter logistic) equation. The fitted midpoints $\left(\mathrm{IC}_{50} \mathrm{~s}\right)$ of the curves were compared statistically using the $\mathrm{F}$ test.

\section{Results}

3.1. Effect of Phenobarbitone and Xylopic Acid High and Low Dose on CYP 1A2. All the treatment groups produced significant $(P<0.05)$ CYP $1 \mathrm{~A} 2$ enzyme induction as shown in Figure 2. Administration of xylopic acid at a high dose of $100 \mathrm{mg} / \mathrm{kg}$ induced CYP $1 \mathrm{~A} 2$ by $81.60 \%$ while low dose of $30 \mathrm{mg} / \mathrm{kg}$ by $77.20 \%$. Phenobarbitone pretreatment induced CYP $1 \mathrm{~A} 2$ by $70.10 \%$ as compared to the nontreatment group that served as the control.

3.2. Effect of Phenobarbitone and Xylopic Acid on CYP 1A1/1A2. All the treatment groups had a significant $(P<0.05)$ effect on CYP 1A1/1A2, with phenobarbitone alone exhibiting the highest enzyme induction with a percentage activity of $139.9 \%$ (Figure 3$)$. A high dose of xylopic acid $(100 \mathrm{mg} / \mathrm{kg}$ ) induced the enzyme resulting in an activity of $106.9 \%$ while xylopic acid low dose induced the enzyme with an activity of $118 \%$ compared to the negative control. The efficacy order for the various treatment groups on CYP $1 \mathrm{~A} 1 / 1 \mathrm{~A} 2$ was phenobarbitone $>$ xylopic acid low dose $>$ xylopic acid high dose.

3.3. Effect of Phenobarbitone and Xylopic Acid on CYP 2B1/2B2. From Figure 4, the phenobarbitone pretreatment group had the most significant $(P<0.001)$ effect on CYP 2B1/2B2. XA low and high doses did not produce a significant effect on CYP 2B1/2B2 compared to the no treatment group. However, there was about a 2.5 -fold increase in CYP 2B1/2B2 activity of phenobarbitone-treated animals compared to both doses of XA.

3.4. Effect of Phenobarbitone and Xylopic Acid on CYP $3 A 4$. Figure 5 illustrates the activity of phenobarbitone, ketoconazole, and XA low and high doses. Phenobarbitone induced CYP 3A4 with an about 6-fold increase in activity, while ketoconazole and XA low and high dose produced about 2- to 3 -fold inhibition of CYP 3A4 activities. Comparatively, the inhibitory activity of XA high dose was approximately 1.36 times more than the standard ketoconazole.

3.5. Effect of Phenobarbitone and Xylopic Acid on CYP 2C9. Figure 6 shows the effect of phenobarbitone and xylopic acid low and high dose on the biotransformation of diclofenac by CYP 2C9. All the treatment groups significantly induced the enzyme activity with percentage enzyme induction of $184.9 \%, 129.9 \%$, and $151.8 \%$ for phenobarbitone, xylopic acid low dose, and xylopic acid high dose, respectively, as compared to the no-treatment group $(P<0.001)$.

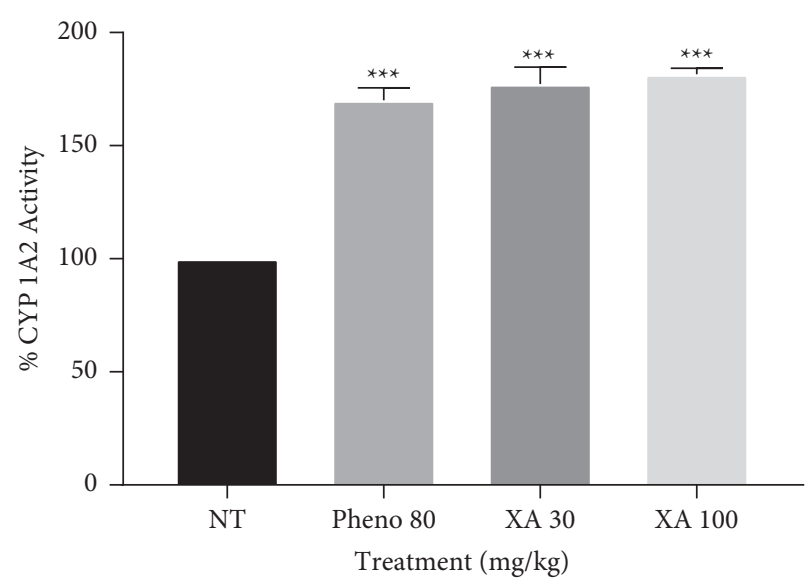

Figure 2: Effect of phenobarbitone (Pheno 80), xylopic acid low dose (30 mg/kg; XA 30), and xylopic acid high dose (100 mg/kg; XA $100)$ on CYP 1A2 (MROD) enzyme activity. Data are presented as mean \pm S.E.M. ${ }^{* * *} P<0.001$ compared to no-treatment group (oneway ANOVA followed by Tukey's post hoc test).

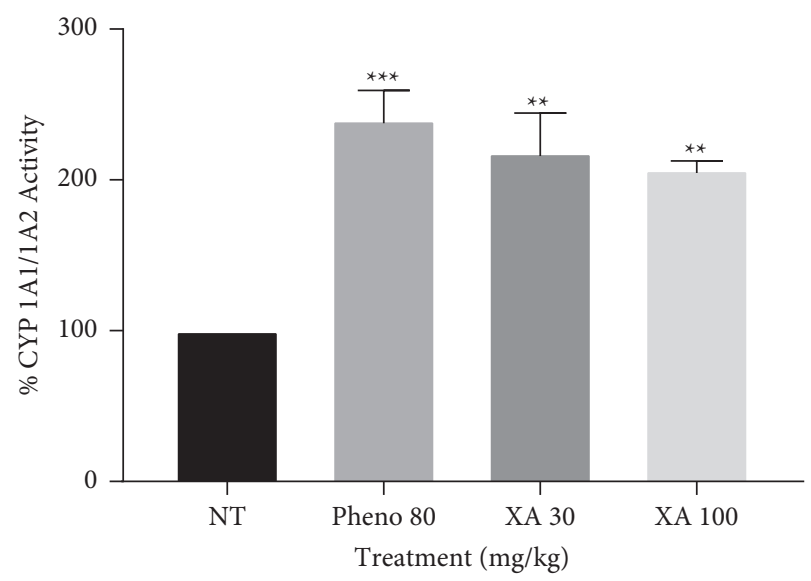

Figure 3: Effect of phenobarbitone (Pheno 80), xylopic acid low dose (30 mg/kg; XA 30), and xylopic acid high dose (100 mg/kg; XA 100) on CYP 1A1/1A2 (EROD) enzyme activity. Data are presented as mean \pm S.E.M. $P<0.01,{ }^{* * *} P<0.001$ compared to the negative control (no treatment) group (one-way ANOVA followed by Tukey's post hoc test).

3.6. Effect of Phenobarbitone and Xylopic Acid on CYP $2 D 6$. Effects of phenobarbitone, XA low dose, and XA high dose on CYP 2D6 are shown in Figure 7. The figure describes a significance in the difference between CYP 2D6 activity of the negative control experiment and the treated $(P<0.001)$ for phenobarbitone and XA high dose and a significance of $(P<0.05)$ in the case of XA low dose. Phenobarbitone, XA low dose, and XA high dose had activities of $48.6 \%, 24 \%$, and $80.5 \%$, respectively. XA high dose was 1.65 times more effective $(P=0.0029)$ than the standard phenobarbitone.

3.7. Effect of Xylopic Acid on CYP $3 A 4$ In Vitro. Figures 8 and 9 show the effect of various concentrations of ketoconazole and xylopic acid on CYP 3A4 in vitro. Ketoconazole was used as the positive control. The $\mathrm{IC}_{50}$ values for ketoconazole 


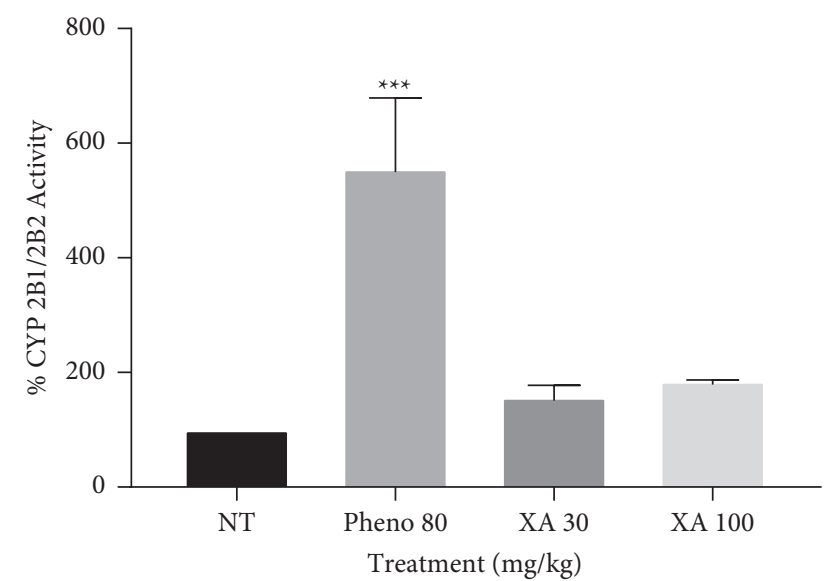

FIgURe 4: Effect of phenobarbitone (Pheno 80), xylopic acid low dose $(30 \mathrm{mg} / \mathrm{kg}$; XA 30), and xylopic acid high dose (100 mg/kg; XA $100)$ on CYP $2 B 1 / 2 B 2$ enzyme. Data are presented as mean \pm S.E.M. ${ }^{* * *} P<0.001$ compared to the no-treatment group (one-way ANOVA followed by Tukey's post hoc test).

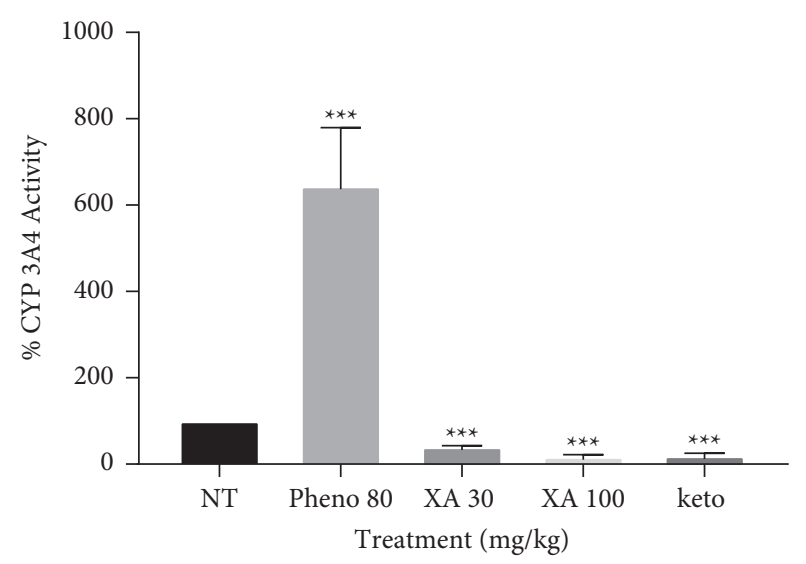

FIGURE 5: Effect of phenobarbitone (Pheno 80), xylopic acid low dose (30 mg/kg; XA 30), and xylopic acid high dose (100 mg/kg; XA 100 ), and ketoconazole (keto) on CYP 3A4 enzyme. Data are presented as mean \pm S.E.M ${ }^{* * *} P<0.001$ compared to the notreatment group (one-way ANOVA followed by Tukey's post hoc test).

and xylopic acid were $0.091 \pm 0.002 \mu \mathrm{M}$ and $1.30 \pm 0.01 \mu \mathrm{M}$, respectively. Thus, ketoconazole showed more potent inhibition of CYP 3A4 than xylopic acid.

\section{Discussion}

The study evaluated the ability of xylopic acid to influence the activity of hepatic CYPs in rats in vitro and in vivo. Interestingly, a recent study suggested that xylopic acid has a biphasic effect on rat hepatic CYP450 enzymes at low and high doses [24], but no specific enzymes were implicated in the biotransformation of the compound. Again, there are no studies on the effect of xylopic acid or the fruit of $X$. aethiopica (which contains xylopic acid) on human hepatic CYP450 enzymes although the fruits are heavily consumed as a spice in local dishes. Therefore, there are no

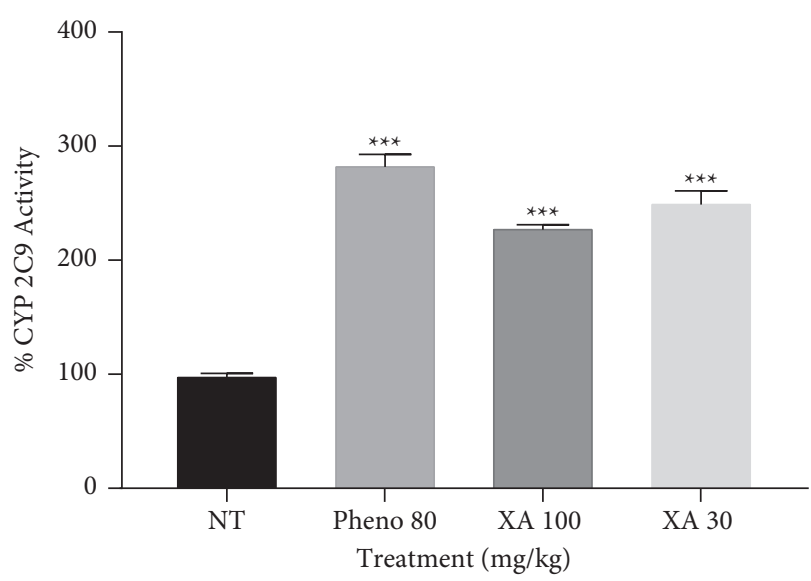

Figure 6: Effect of phenobarbitone (Pheno 80), xylopic acid low dose (30 mg/kg; XA 30), and xylopic acid high dose (100 mg/kg; XA 100) on CYP 2C9 (diclofenac hydroxylation). Data are presented as mean \pm S.E.M ${ }^{* * *} P<0.001$ compared to the no-treatment group (one-way ANOVA followed by Tukey's post hoc test).

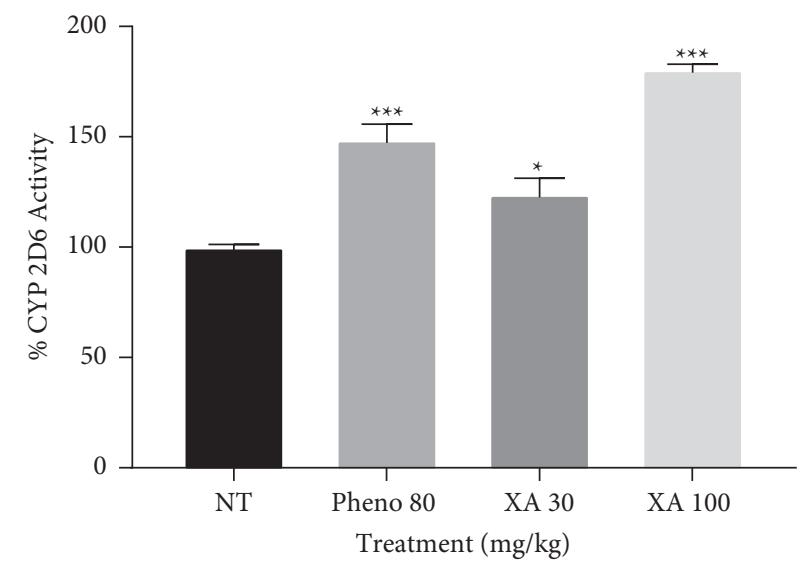

Figure 7: Effect of phenobarbitone (Pheno 80), xylopic acid low dose (30 mg/kg; XA 30), and xylopic acid high dose (100 mg/kg; XA 100) on CYP 2D6 (dextromethorphan O-demethylation). Data are presented as mean \pm S.E.M. ${ }^{*} P<0.05$ and ${ }^{* * *} P<0.001$ compared to the no-treatment group (one-way ANOVA followed by Tukey's post hoc test).

data on the metabolism of xylopic acid or its related kaurene diterpenes in rodents or humans, hence limiting these studies to implicate any possible metabolite of xylopic acid on the effects observed in these studies. Owing to the fact that $X$. aethiopica is heavily consumed, xylopic acid or its possible active metabolites can reach concentrations high enough to interact with other medications when taken concurrently. It was therefore imperative to identify the specific isoenzymes induced and inhibited on the administration of xylopic acid with a focus on CYP 1A1/1A2, 1A2, 2B1/2B2, 3A4, 2D6, and 2C9.

Xylopic acid induced CYP 1A1/1A2 at high and low doses. Other substances that induce CYP $1 \mathrm{~A} 1 / 1 \mathrm{~A} 2$ are barbiturates, tobacco, and rifampin among others [27].

Xylopic acid induced CYP $1 \mathrm{~A} 2$ in vivo at low and high doses, like the effect seen in phenobarbitone. Studies have 


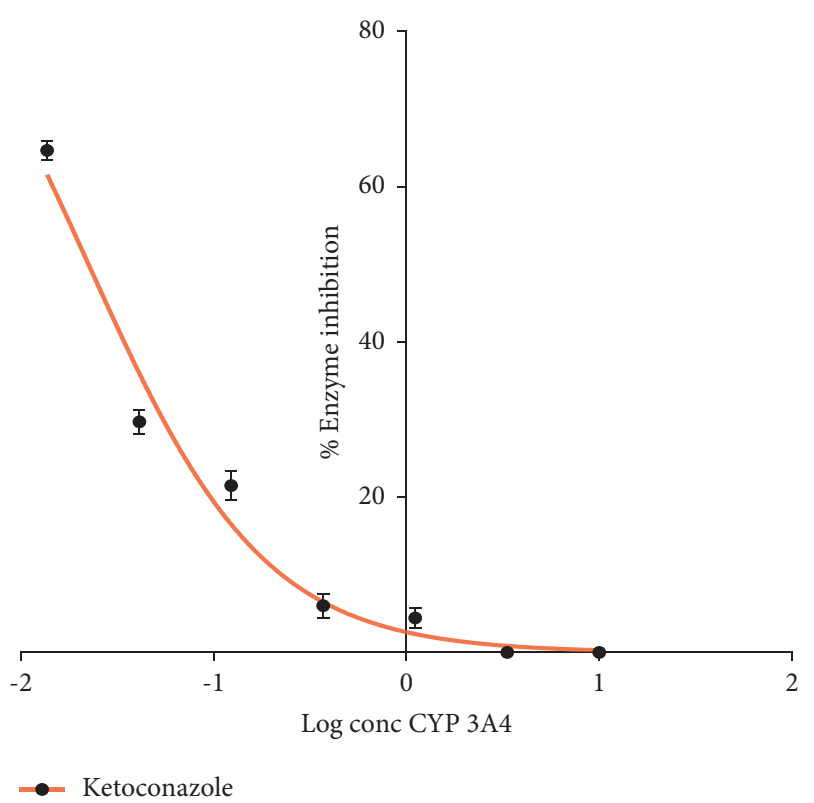

FIGURE 8: Dose-response curve of ketoconazole on the inhibition of CYP 3A4. Ketoconazole was used as the standard drug and various concentrations were used to determine the $\mathrm{IC}_{50}$ values. Data are presented as mean \pm S.E.M.

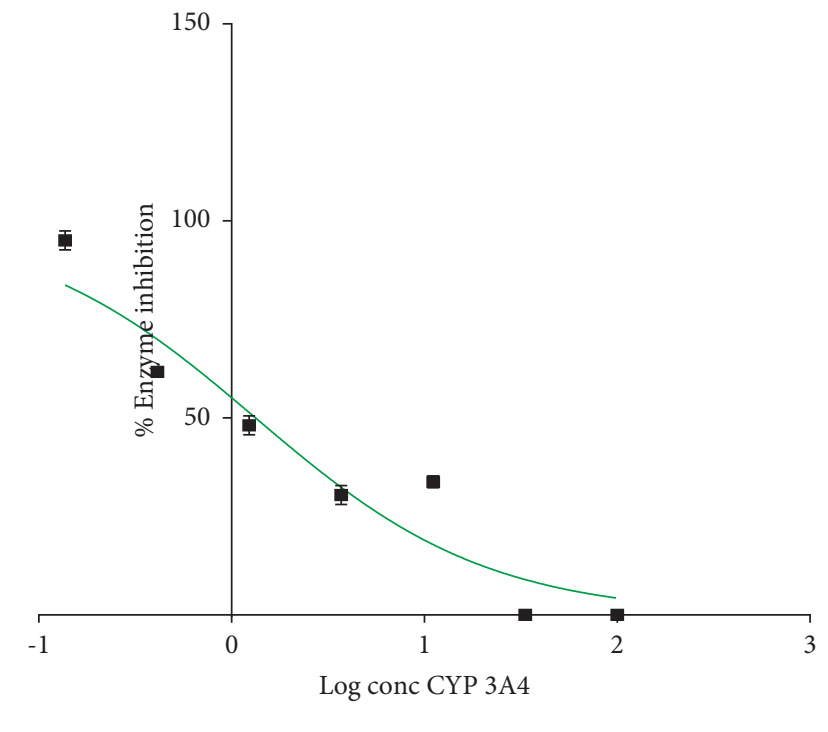

$-\mathrm{XA}$

FIGURE 9: Dose-response curve of varying concentrations of XA against enzyme inhibition on CYP 3A4. XA was added at various concentrations to determine the $\mathrm{IC}_{50}$ value. Data are presented as mean \pm S.E.M. Nonlinear regression analysis was used in plotting the curve.

shown that CYP $1 \mathrm{~A} 2$ is responsible for the primary metabolism of theophylline and propranolol amongst others [28]. A study implicated cimetidine which is a histamine $\mathrm{H}_{2}$-receptor antagonist as an inhibitor of CYP $1 \mathrm{~A} 2$ [28]. This suggested that the administration of xylopic acid with $\mathrm{H}_{2}$-receptor antagonists may result in drug-drug interaction.
CYP 2B1/2B2 is a phenobarbital-inducible member of the P450 xenobiotic-inducible superfamily involved in the hydroxylation of decanoic and other fatty acids [29]. The current study revealed that xylopic acid had no activity (induction/inhibition) on CYP 2B1/2B2.

CYP 3A4 was inhibited by low and high doses of xylopic acid. The inhibition observed was weaker than that expressed by ketoconazole, a standard inhibitor of 3A4. CYP 3A4 accounts for 30-50\% of drugs metabolized through type I enzymes [30]; therefore, it was paramount to investigate the effect of xylopic acid on CYP 3A4. Although the dose of xylopic acid used in the in vivo studies were 30 and $100 \mathrm{mg} / \mathrm{kg}$, the amount absorbed into the bloodstream was not measured, but the concentration achieved in the blood can be high enough to produce food-drug interaction since this concentration was able to inhibit the CYP $3 \mathrm{~A} 4$ enzymes.

Xylopic acid at low and high doses induced CYP 2C9. It is primarily expressed in the liver; a study suggests that its expression level is the second highest among CYP isoforms. It is responsible for the metabolic clearance of up to $15-20 \%$ of all drugs [31]. Some other substrates metabolized by this CYP aside from diclofenac include alkylating agents such as cyclophosphamide [32] and antiestrogenic drugs such as tamoxifen [33]. Treatment with rifampicin has been implicated in increased clearance of drugs metabolized by CYP 2C9 [32]. Drugs that inhibit this enzyme include fluconazole and sulfaphenazole. There is evidence supporting the danger of drug-drug interaction when sulfaphenazole is added to a therapeutic regime that includes drugs with a low therapeutic index, such as warfarin [34]. This suggests that the use of xylopic acid with these inhibitors should be carefully considered.

Patients vary widely in their response to drugs. They include individuals with no CYP 2D6 activity and individuals with genetically elevated CYP 2D6 activity, suggesting an altered response to drugs that are CYP 2D6 substrates. Drugs that inhibit CYP 2D6 activity are likely to increase the plasma concentrations of other medications, such as fluoxetine and paroxetine leading to drug toxicity [35].

\section{Conclusion}

Xylopic acid induced rat liver microsomal CYP 1A1/1A2, 1A2, 2D6, and 2C9; inhibited CYP 3A4; and did not affect CYP 2B1/2B2. Since these enzymes are implicated in the metabolism of several drugs, it is expected that XA can potentially influence the metabolism of other drugs through food-drug and drug-drug interactions.

\section{Data Availability}

The data in support of the findings of this study may be requested and obtained from the corresponding author.

\section{Conflicts of Interest}

The authors declare they have no conflicts of interest. 


\section{Authors' Contributions}

RAO, EOA, EO, and RPB were involved in the conceptualization of the study, design of the study, data analysis, and proofreading of the manuscript. MAP, EAA, ADF, and SAO were involved in the execution of the experiments, collection of the plant materials, data analysis, and drafting of the manuscript.

\section{Acknowledgments}

This manuscript was drafted from studies conducted in a thesis (Antiplasmodial activity and cytochrome p450-mediated pharmacokinetic drug interactions of xylopic acid) submitted to the University of Cape Coast. The authors thank the staff and fellows of NMIMR, Departments of Clinical Pathology, Virology, and Animal Experimentation. The authors also appreciate Mr. Jeremiah Aderoju and all laboratory technologists of the School of Pharmacy and Pharmaceutical Sciences, UCC.

\section{References}

[1] D. Singh, R. Gupta, and S. A. Saraf, "Herbs-are they safe enough? An overview," Critical Reviews in Food Science and Nutrition, vol. 52, no. 10, pp. 876-898, 2012.

[2] B. Yang, Y. Xie, M. Guo, M. H. Rosner, H. Yang, and C. Ronco, "Nephrotoxicity and Chinese herbal medicine," Clinical Journal of the American Society of Nephrology, vol. 13, no. 10, pp. 1605-1611, 2018.

[3] R. P. Biney, C. K. Benneh, E. O. Ameyaw, E. Boakye-Gyasi, and E. Woode, "Xylopia aethiopica fruit extract exhibits antidepressant-like effect via interaction with serotonergic neurotransmission in mice," Journal of Ethnopharmacology, vol. 184, no. 1, pp. 49-57, 2016.

[4] H. Yuan, Q. Ma, L. Ye, and G. Piao, "The traditional medicine and modern medicine from natural products," Molecules, vol. 21, no. 5, p. 559, 2016.

[5] E. N. Oge, E. Obese, R. P. Biney et al., "A review of pharmacological effects of xylopic acid," International Journal of Basic \& Clinical Pharmacology, vol. 9, no. 5, pp. 813-818, 2020.

[6] A. M. Towns and T. Van Andel, "Wild plants, pregnancy, and the food-medicine continuum in the southern regions of Ghana and Benin," Journal of Ethnopharmacology, vol. 179, no. 1, pp. 375-382, 2016.

[7] H. C. Martin, Antibacterial Activity of Piper Guineense, Xylopia Aethiopica, and Allium cepa against Bacteria Isolated from Spoilt Soup Preparations, Doctoral dissertation, University of Nigeria, Nigeria, 2017.

[8] S. Adefeghaa, G. Oboha, T. Odubanjoa, and O. Ogunsuyia, "A comparative study on the antioxidative activities, anticholinesterase properties, and essential oil composition of clove (Syzygium aromaticum) bud and Ethiopian pepper (Xylopia aethiopica)," Riv. Ital. Sost. Gr, vol. 92, no. 1, pp. 257-268, 2015.

[9] T. Fleischer, M. Mensah, A. Mensah, G. Komlaga, S. Gbedema, and H. Skaltsa, "Antimicrobial activity of essential oils of Xylopia aethiopica," African Journal of Traditional, Complementary and Alternative Medicines, vol. 5, no. 4, pp. 391-393, 2008.

[10] E. O. Nwaichi and P. Anyanwu, "Effect of heat treatment on the antioxidant properties of Tetrapleura tetraptera, Xylopia aethiopica and Piper guineense," Journal of Medical Research and Development (JMRD), vol. 2, no. 3, pp. 59-63, 2013.

[11] D. M. Johnson and N. A. Murray, "A revision of Xylopia L. (Annonaceae): the species of tropical Africa," PhytoKeys, vol. 97, no. 1, p. 1, 2018.

[12] R. Ebana, U. Ekanemesang, U. Edet, and E. Omoruyi, "Phytochemical screening and antimicrobial activity of Xylopia aethiopica and Gongronema latifolium on common pathogens," Journal of Advances in Biology \& Biotechnology, vol. 9, no. 4, pp. 1-7, 2016.

[13] B. A. Kouame, J. A. Mamyrbekova-Bekro, J. Nemlin, and B. Yves-Alain, "Chemical composition and antioxidant activities of essential oils of Xylopia aethiopica (Dunal) a. rich," European Journal of Scientific Research, vol. 37, no. 2, pp. 311-318, 2009.

[14] E. O. Ameyaw, Anti-Nociceptive Properties of the Ethanolic Extract of Fruits of Xylopia Aethiopica (Dunal) A. Rich (Annonaceae) and its Major Constituent, Xylopic Acid, Doctoral dissertation, Kwame Nkrumah University of Science and Technology, Kumasi, Ghana, 2012.

[15] E. Woode, E. Ameyaw, E. Boakye-Gyasi, and W. M. Abotsi, "Analgesic effects of an ethanol extract of the fruits of Xylopia aethiopica (Dunal) A. Rich (Annonaceae) and the major constituent, xylopic acid in murine models," Journal of Pharmacy and BioAllied Sciences, vol. 4, no. 4, p. 291, 2012.

[16] L. I. Somova, F. O. Shode, K. Moodley, and Y. Govender, "Cardiovascular and diuretic activity of kaurene derivatives of Xylopia aethiopica and Alepidea amatymbica," Journal of Ethnopharmacology, vol. 77, no. 2-3, pp. 165-174, 2001.

[17] E. Ekuadzi, R. P. Biney, C. K. Benneh, B. Osei Amankwaa, and J. Jato, "Antiinflammatory properties of betulinic acid and xylopic acid in the carrageenan-induced pleurisy model of lung inflammation in mice," Phytotherapy Research, vol. 32, no. 3, pp. 480-487, 2018.

[18] J. N. Boampong, E. O. Ameyaw, B. Aboagye et al., "The curative and prophylactic effects of xylopic acid on Plasmodium berghei infection in mice," Journal of Parasitology Research, vol. 2013, Article ID 356107, 7 pages, 2013.

[19] A. E. Ofori, W. Eric, K. Samuel, B. P. Robert, and B. J. Nyarko, "Anti-nociceptive synergism of pregabalin and xylopic acid Co-administration in paclitaxel induced neuropathy: isobolographic analysis," Pharmacognosy Journal, vol. 7, no. 6, pp. 363-368, 2015.

[20] R. Appiah-Opong, J. N. M. Commandeur, E. Istyastono, J. J. Bogaards, and N. P. E. Vermeulen, "Inhibition of human glutathioneS-transferases by curcumin and analogues," Xenobiotica, vol. 39, no. 4, pp. 302-311, 2009.

[21] J. M. Nguta, R. Appiah-Opong, A. K. Nyarko, D. YeboahManu, and P. G. A. Addo, "Medicinal plants used to treat TB in Ghana," International Journal of Mycobacteriology, vol. 4, no. 2, pp. 116-123, 2015.

[22] R. Appiah-Opong, N. A. Ankrah, A. K. Nyarko, and M. Ofosuhene, "Phytomedicines: safety and efficacy studies on Ghanaian medicinal plants," Towards Effective Disease Control in Ghana: Research and Policy Implications: Volume 2 Other Infectious Diseases and Health Systems, vol. 2, University of Ghana, Accra, Ghana, 2014.

[23] Z. Hu, X. Yang, P. C. L. Ho et al., "Herb-drug interactions," Drugs, vol. 65, no. 9, pp. 1239-1282, 2005.

[24] R. P. Biney, P. K. Mantel, E. Boakye-Gyasi, K. E. Kukuia, and E. Woode, "Neuropharmacological effects of an ethanolic fruit extract of Xylopia aethiopica and xylopic acid, a kaurene diterpene isolate, in mice," West African Journal of Pharmacy, vol. 25, no. 1, pp. 106-107, 2014. 
[25] H. J. Harwood Jr, S. F. Petras, L. D. Shelly et al., "Isozymenonselective $\mathrm{N}$-substituted bipiperidylcarboxamide acetylCoA carboxylase inhibitors reduce tissue malonyl-CoA concentrations, inhibit fatty acid synthesis, and increase fatty acid oxidation in cultured cells and in experimental animals," Journal of Biological Chemistry, vol. 278, no. 39, pp. 3709937111, 2003.

[26] B. B. N'guessan, S. K. Amponsah, G. J. Dugbartey et al., "In vitro antioxidant potential and effect of a glutathione-enhancer dietary supplement on selected rat liver cytochrome P450 enzyme activity," Evidence-based Complementary and Alternative Medicine, vol. 2018, Article ID 7462839, 8 pages, 2018.

[27] M. Martignoni, G. M. M. Groothuis, and R. de Kanter, "Species differences between mouse, rat, dog, monkey and human CYP-mediated drug metabolism, inhibition and induction," Expert Opinion on Drug Metabolism and Toxicology, vol. 2, no. 6, pp. 875-894, 2006.

[28] C. Martínez, C. Albet, J. A. Agúndez, E. Herrero, J. A. Carrillo, and M. Márquez, "Comparative in vitro and in vivo inhibition of cytochrome P450 CYP1A2, CYP2D6, and CYP3A by H2receptor antagonists," Clinical Pharmacology \& Therapeutics, vol. 65, no. 4, pp. 369-376, 1999.

[29] B. Meunier, S. P. De Visser, and S. Shaik, "Mechanism of oxidation reactions catalyzed by cytochrome P450 enzymes," Chemical Reviews, vol. 104, no. 9, pp. 3947-3980, 2004.

[30] A. Haddad, M. Davis, and R. Lagman, "The pharmacological importance of cytochrome CYP3A4 in the palliation of symptoms: review and recommendations for avoiding adverse drug interactions," Supportive Care in Cancer, vol. 15, no. 3, pp. 251-257, 2007.

[31] Y. Pan, K. H. Tiong, B. A. Abd-Rashid et al., "Effect of eurycomanone on cytochrome P450 isoforms CYP1A2, CYP2A6, CYP2C8, CYP2C9, CYP2C19, CYP2E1 and CYP3A4 in vitro," Journal of Natural Medicines, vol. 68, no. 2 , pp. 402-406, 2014.

[32] D. Van Booven, S. Marsh, H. McLeod et al., "Cytochrome P450 2C9-CYP2C9," Pharmacogenetics and Genomics, vol. 20 , no. 4, pp. 277-281, 2010.

[33] J. Chen and K. Raymond, "Roles of rifampicin in drug-drug interactions: underlying molecular mechanisms involving the nuclear pregnane X receptor," Annals of Clinical Microbiology and Antimicrobials, vol. 5, no. 1, pp. 3-11, 2006.

[34] E. Tanaka, "Clinically important pharmacokinetic drug-drug interactions: role of cytochrome P450 enzymes," Journal of Clinical Pharmacy and Therapeutics, vol. 23, no. 6, pp. 403416, 1998.

[35] C. Kawanishi, S. Lundgren, H. Agren, and L. Bertilsson, "Increased incidence of CYP2D6 gene duplication in patients with persistent mood disorders: ultrarapid metabolism of antidepressants as a cause of nonresponse. A pilot study," European Journal of Clinical Pharmacology, vol. 59, no. 11, pp. 803-807, 2004. 